\title{
Substance $P$ accelerates wound healing in type 2 diabetic mice through endothelial progenitor cell mobilization and Yes-associated protein activation
}

\author{
JIHYUN UM ${ }^{1}$, JINYEONG YU ${ }^{1}$ and KI-SOOK PARK ${ }^{2}$ \\ ${ }^{1}$ Graduate School of Biotechnology, Kyung Hee University, Yongin, Gyeonggi 17104; \\ ${ }^{2}$ East-West Medical Research Institute, Kyung Hee University, Seoul 02447, Republic of Korea
}

Received March 10, 2016; Accepted February 16, 2017

DOI: $10.3892 / \mathrm{mmr} .2017 .6344$

\begin{abstract}
Wound healing is delayed in diabetes due to a number of factors, including impaired angiogenesis and poor dermal healing. The present study demonstrated that subcutaneous administration of substance $\mathrm{P}(\mathrm{SP})$ accelerates wound healing in $\mathrm{db} / \mathrm{db}$ type 2 diabetic mice (db/db mice). SP injection (10 nM/kg, subcutaneously) enhanced angiogenesis, induced the mobilization of endothelial progenitor cells (EPCs) and increased the number of EPC-colony forming units (EPC-CFUs) in the bone marrow of $\mathrm{db} / \mathrm{db}$ mice. Immunohistochemistry was performed to check the effects of SP on the cellular proliferation and the subcellular localization of Yes-associated protein (YAP) in the wound dermis. SP also upregulated cellular proliferation in the injured dermis of $\mathrm{db} / \mathrm{db}$ mice. Compared with the control group, an increased number of cells in the wound dermis of SP-treated mice exhibited nuclear localization of YAP, which induces cellular proliferation. The results of the current study indicate that subcutaneous administration of SP may be a promising therapeutic strategy to treat diabetic wounds exhibiting impaired angiogenesis and dysfunctional dermal wound healing.
\end{abstract}

\section{Introduction}

Wound healing is an essential biological process in the maintenance of tissue and organ homeostasis (1). Various events

Correspondence to: Professor Ki-Sook Park, East-West Medical Research Institute, Kyung Hee University, 26 Kyungheedae-ro, Dongdaemun-gu, Seoul 02447, Republic of Korea

E-mail:kisookpark@khu.ac.kr

Abbreviations: ECM, extracellular matrix; EPC, endothelial progenitor cell; EPC-CFU, endothelial progenitor cell-colony forming unit; $\alpha$ SMA, $\alpha$-smooth muscle actin; SP, substance P; YAP, Yes-associated protein

Key words: substance $\mathrm{P}$, wound, diabetes, endothelial progenitor cells, Yes-associated protein are regulated during wound healing, including inflammation, angiogenesis, cell proliferation, cell migration, cell death and the synthesis and reorganization of extracellular matrix (ECM) $(1,2)$. Wound healing is delayed in chronic conditions such as diabetic wounds, and defects in multiple processes associated with the wound healing process are responsible for this delay (3). For example, angiogenesis and dermal wound healing are dependent upon the proliferation and migration of dermal cells and ECM accumulation, and these processes are severely impaired in diabetic wound healing (3).

In response to various factors, endothelial progenitor cells (EPCs) are mobilized and recruited by injured tissues, where they differentiate into endothelial cells and induce new blood vessel growth to accelerate wound healing and regeneration (4-6). Compared with the normal injury response, the mobilization and recruitment of EPCs are impaired in diabetic wounds, and reduced levels of stromal cell-derived factor- 1 at the wound may be implicated in this impairment $(7,8)$.

The Hippo signaling pathway regulates various important biological phenomena, including cell proliferation, cell death, cell polarity and mechanotransduction $(9,10)$. The Yes-associated protein (YAP) is one of the terminal effectors in the Hippo pathway and it regulates the transcription of target genes in the nuclei by interacting with the transcriptional enhancer associated domain family of transcription factors (9). YAP activity is primarily regulated by subcellular localization following phosphorylation (11). When the Hippo signaling pathway is activated, YAP is phosphorylated by upstream kinases, large tumor suppressor kinase 1 (LATS1) and LATS2, and the phosphorylated YAP is retained in the cytoplasm via physical interaction with 14-3-3 proteins (11). However, unphosphorylated YAP enters the nucleus and activates target genes that induce cell proliferation (9-11). Wound healing requires YAP activation in epithelial and dermal tissues (12).

Substance P (SP) is a peptide composed of 11 amino acids that was identified as a neurotransmitter in the central nervous system associated with pain sensation. It has been also demonstrated that SP acts as an immune modulator and injury messenger in various peripheral tissues (13). Furthermore, SP mobilizes mesenchymal stem cells (13) and EPCs (6) in the bone marrow, and induces them to migrate into the injured 
peripheral tissues where they are involved in regeneration. It has also been demonstrated that SP accelerates the normal acute and chronic wound healing processes (14-16). Notably, a previous study demonstrated that subcutaneous administration of SP accelerates the normal acute wound healing response via increased angiogenesis resulting from SP-mediated EPC mobilization (17). By contrast, serum levels of SP are decreased in diabetic patients (18), and the SP degradation activity of neutral endopeptidase is increased in chronic diabetic wounds (19). These results indicate that the decrease in SP may be implicated in impaired diabetic wound healing.

The present study used db/db type 2 diabetic (db/db) mice to determine whether subcutaneous administration of SP accelerates healing in an acute diabetic wound model. In addition, the current study also investigated whether impaired EPC mobilization in diabetic wounds could be rescued in $\mathrm{db} / \mathrm{db}$ mice through the subcutaneous administration of SP. Furthermore, the present study investigated whether YAP activation was involved in the SP-mediated acceleration of diabetic dermal wound healing.

\section{Materials and methods}

Mice. A total of 9 male $\mathrm{db} / \mathrm{db}$ mice (7-17 weeks-old; 20-25 g) were purchased from Nara Biotech (Seoul, Korea) and were maintained under a $12 \mathrm{~h}$ light:dark cycle at a controlled temperature $\left(25 \pm 2^{\circ} \mathrm{C}\right)$ in a humidified atmosphere $(40-70 \%)$ with unlimited access to food and water. All procedures were approved by the Kyung Hee University Medical Center Institutional Animal Care and Use Committee (Seoul, Korea). The mice were randomly divided into two groups; 4 mice for a control group and 5 mice for the SP treated group.

Wound healing model. After hyperglycemia was confirmed in 16-week-old db/db mice (484-600 mg/dl), full-thickness skin wounds were created. Mice were anesthetized using ketamine (8 mg/kg; Yuhan Co., Ltd., Seoul, Korea) and Rompun ${ }^{\circledR}$ (Xylazine; $5.6 \mathrm{mg} / \mathrm{kg}$; Bayer Korea Ltd.; Bayer AG, Leverkusen, Germany). The dorsal skin was shaved and a wound was created using a $4 \mathrm{~mm}$ biopsy punch. Hydrogel (Pluronic F127; Sigma-Aldrich; Merck KGaA, Darmstadt, Germany) was applied to the wounds. Subsequently, the wounds were dressed with Mepitel ${ }^{\circledR}$ (Mölnlycke Health Care, Gothenburg, Sweden) and Tegaderm $^{\text {TM }}$ (3M; Maplewood, MN, USA). Substance P (10 nM/kg; EMD Millipore, Billerica, MA, USA) was subcutaneously injected once a day into the flanks of the mice for 2 consecutive days after wound creation. Digital images of the wounds were obtained at 7 days and wound size was analyzed using Adobe Photoshop CS6 software (Adobe Systems, Inc., San Jose, CA, USA). The animals were sacrificed by cervical dislocation and the wound tissues were harvested for further analysis.

Tissue processing and histological analysis. Harvested wound tissues were collected and fixed overnight in $4 \%$ paraformaldehyde (PFA; $3 \mathrm{M}$ ) at $4^{\circ} \mathrm{C}$. The fixed tissues were embedded in paraffin according to standard procedures and the tissue paraffin blocks were sectioned at a thickness of $4 \mu \mathrm{m}$. To perform histological analysis, the sections were stained with hematoxylin and eosin (Sigma-Aldrich; Merck KGaA). Images were collected using an Olympus BX41 light microscope (Olympus Corporation, Tokyo, Japan).

Immunohistochemistry. Immunostaining against $\alpha$-smooth muscle actin ( $\alpha$ SMA), proliferating cell nuclear antigen (PCNA) and YAP was performed according to standard procedures. Briefly, paraffin sections $(4 \mu \mathrm{m})$ were deparaffinized and dehydrated. The dehydrated sections were subsequently incubated in peroxidase blocking solution $(0.5 \%$ $\mathrm{H}_{2} \mathrm{O}_{2}$ in methanol) to inhibit endogenous peroxidase activity and antigen retrieval was performed by boiling in sodium citrate buffer (10 mM; pH 6.0). The sections were blocked with 5\% nonfat milk (BD Biosciences, Franklin Lakes, NJ, USA) in phosphate-buffered saline containing $0.2 \%$ Tween-20 (PBST) for $30 \mathrm{~min}$ at room temperature. Then, the sections were incubated in PBST containing 5\% nonfat milk for $1.5 \mathrm{~h}$ using the following primary antibodies: Anti- $\alpha$ SMA (M0851; 1:200; Dako; Agilent Technologies, Inc., Santa Clara, CA, USA), anti-PCNA (sc-56; 1:50; Santa Cruz Biotechnology, Inc., Dallas, TX, USA) and anti-YAP (14,074; 1:100; Cell Signaling Technology, Inc., Danvers, MA, USA). Non-fluorescent staining was performed using the VECTASTAIN ${ }^{\circledR}$ Elite $^{\circledR}$ ABC kit (Vector Laboratories, Inc., Burlingame, CA, USA) and the DAB peroxidase (HRP) substrate kit (Vector Laboratories, Inc.), according to the manufacturer's protocols. Nuclei were stained in pink with Vector ${ }^{\circledR}$ Nuclear Fast Red (Vector Laboratories, Inc.) or purple with hematoxylin. Images were collected using an Olympus BX41 light microscope (Olympus Corporation, Tokyo, Japan). If required, the total tissue area in each image was measured using Adobe Photoshop CS6 software (Adobe Systems, Inc.).

In vivo Matrigel plug assay and EPC-colony forming unit $(C F U)$ assay. Following anesthetization, $0.2 \mathrm{ml}$ growth factor-reduced Matrigel (BD Biosciences, Franklin Lakes, NJ, USA) combined with heparin (40 U/ml; Sigma-Aldrich; Merck $\mathrm{KGaA})$ and murine basic fibroblast growth factor $(0.5 \mu \mathrm{g} / \mathrm{ml}$; R\&D Systems, Inc., Minneapolis, MN, USA) was injected subcutaneously into the dorsal skin of 11-week-old $\mathrm{db} / \mathrm{db}$ mice (301-458 mg/dl). For mice assigned to the SP-treatment group, SP $(10 \mathrm{nM} / \mathrm{kg})$ was injected subcutaneously into the flank for 2 days after injection of the Matrigel mixture. Matrigel plugs were harvested 7 days after injection and fixed in $4 \%$ PFA for $4 \mathrm{~h}$ on ice. After preparing cryosections $(12 \mu \mathrm{m})$, the sections were blocked with $5 \%$ nonfat milk (BD Biosciences) in PBST for $30 \mathrm{~min}$ at room temperature. Then the sections were treated with the secondary antibody (goat anti-rat Alexa Fluor $^{\circledR}$ 488-conjugated IgG; A-11006; 1:500; Invitrogen; Thermo Fisher Scientific, Inc.) for $45 \mathrm{~min}$ at room temperature following the incubation with primary antibody (rat anti-CD31 monoclonal antibody; CBL1337; 1:100; Chemicon; EMD Millipore, Billerica, MA, USA) for $2 \mathrm{~h}$ at room temperature to analyze endothelial cells in the sections. Images were captured using a Zeiss LSM 700 confocal microscope (Zeiss AG, Oberkochen, Germany). Following retrieval of the Matrigel plugs, bone marrow cells obtained from the femur of each mouse were flushed with $\alpha$-minimal essential media (Sigma-Aldrich; Merck KGaA) and 20\% heat-inactivated fetal bovine serum (Invitrogen; Thermo Fisher Scientific, Inc.) (20), and a cell suspension $\left(1 \times 10^{5}\right.$ cells $\left./ \mathrm{ml}\right)$ was made with medium 
A

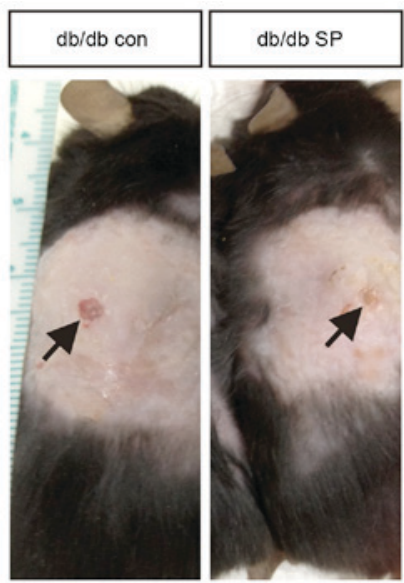

B

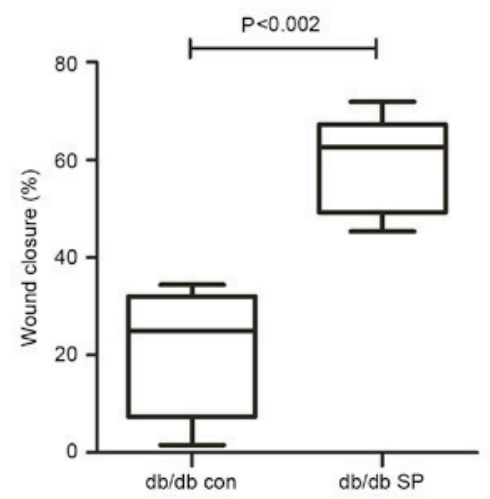

C

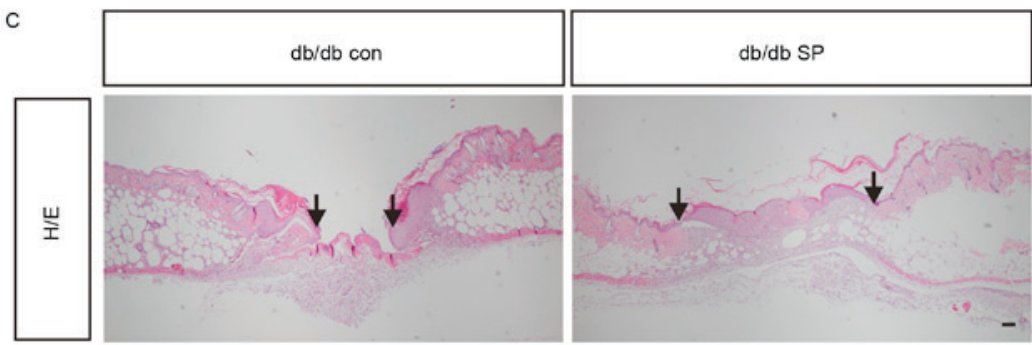

D

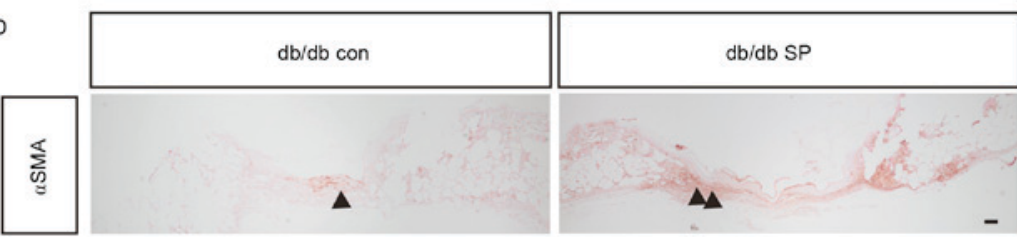

Figure 1. SP-enhanced wound contraction. (A) Representative images of wounds in the db/db con and db/db SP groups 7 days after wound creation. Arrows indicate the wound locations. (B) Wound closure of $\mathrm{db} / \mathrm{db}$ con $(\mathrm{n}=4)$ and $\mathrm{db} / \mathrm{db} \mathrm{SP}$ groups $(\mathrm{n}=5)$. Data are presented as the mean \pm standard deviation. (C) $\mathrm{H} / \mathrm{E}$ staining of wound tissue in $\mathrm{db} / \mathrm{db}$ con and db/db SP groups. Representative results are presented; arrows indicate borders between re-epithelialized and non-epithelialized wound surfaces. (D) Immunohistochemistry of $\alpha$ SMA, representative results are presented. Arrow heads indicate $\alpha$ SMA-positive signals in the dermis. Scale bar, $100 \mu \mathrm{m}$. SP, substance P; db/db con, control diabetic mice; db/db SP, SP-treated diabetic mice; H/E, hematoxylin and eosin; $\alpha \mathrm{SMA}$, $\alpha$-smooth muscle actin.

prepared using the endothelial Cell Growth Medium EGM ${ }^{\mathrm{TM}}-2$ Bulletkit (Lonza Group AG, Basel, Switzerland). Cells were seeded on $35 \mathrm{~mm}$ tissue culture dishes at a density of $1 \times 10^{4}$ cells/dish using MethoCult ${ }^{\mathrm{TM}}$ media (Stemcell Technologies, Inc., Vancouver, BC, Canada) and three dishes were prepared for each mouse. At 9 days after seeding, the cell culture media were carefully washed out and the number of adherent colonies was analyzed using a Nikon ECLIPSE TS 100 inverted microscope (Nikon Corporation, Tokyo, Japan).

Statistical analysis. Data are presented as the mean \pm standard deviation. Student's t-test was used to evaluate differences between sample means. $\mathrm{P}<0.05$ was considered to indicate a statistically significant difference. All statistical analyses were performed using GraphPad Prism 5.01 (GraphPad Software, Inc., La Jolla, CA, USA.).

\section{Results}

SP accelerates wound closing in db/db type 2 diabetic mice. A previous study demonstrated that subcutaneous injection of SP accelerates the wound healing process in normal mice (17). Therefore, the present study investigated whether subcutaneous administration of SP $(10 \mathrm{nM} / \mathrm{kg})$ also affects wound healing in a db/db type 2 diabetic mouse model. At 7 days after wound creation, full-thickness closure of the excision wound was significantly enhanced in $\mathrm{db} / \mathrm{db}$ mice with SP treatment $(59.06 \pm 18.62 \%$; $=6)$ compared with control mice $(21.48 \pm 6.99 \%$; $n=4$; Fig. $1 \mathrm{~A}$ and $\mathrm{B})$. In addition to wound contraction, increased re-epithelialization was observed in the SP-treated mice compared with control mice (Fig. 1C). aSMA-positive myofibroblasts have an important role in wound contracture (21), and the detection of $\alpha$ SMA-positive cells is delayed in diabetic wounds compared with non-diabetic wounds (22). Notably, SP injection increased the number of $\alpha \mathrm{SMA}$-positive cells in wounds of SP-treated mice compared with the control group (Fig. 1D), thereby enhancing wound contraction. Collectively, the results indicate that subcutaneous injection of SP may accelerate wound closure by increasing the number of aSMA-expressing myofibroblasts in $\mathrm{a} \mathrm{db} / \mathrm{db}$ mouse model of type 2 diabetes. 

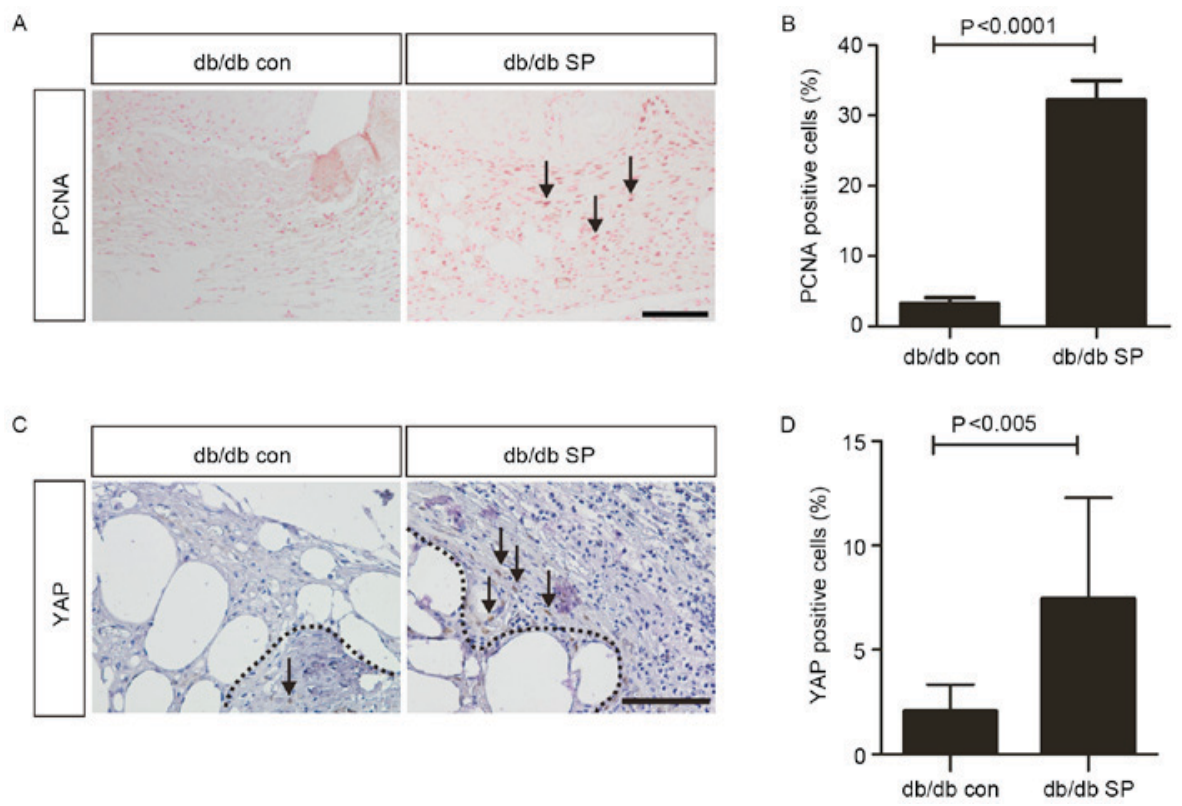

Figure 2. SP-enhanced cellular proliferation and YAP activation. (A) Immunohistochemistry analysis of PCNA, arrows indicate PCNA-positive cells in the dermis of $\mathrm{db} / \mathrm{db}$ con and db/db SP groups. (B) Quantitative analysis of dermal cells expressing PCNA. (C) Immunohistochemistry analysis of YAP, arrows indicate dermal cells expressing YAP in the nuclei. (D) Quantitative analysis of YAP-positive dermal cell nuclei. The percentage represents the number of PCNA-positive dermal cells or YAP-positive dermal cell nuclei in a random wound tissue section image divided by the total number of dermal cell nuclei. Results are presented as the mean + standard deviation. Black, broken lines indicate wound edges. Scale bars, $100 \mu \mathrm{m}$. SP, substance P; YAP, Yes-associated protein; PCNA, proliferating cell nuclear antigen; db/db con, control diabetic mice; db/db SP, SP-treated diabetic mice.

SP enhances cellular proliferation and YAP activation in the wound dermis. YAP is one of the key effectors in the Hippo signaling pathway that controls various important biological phenomena, including cellular proliferation, cell survival and organ size determination $(9,23)$. Notably, it has been previously demonstrated that YAP controls skin wound healing (12). Post-translational stability and nuclear localization of YAP are required for it to function as a transcriptional activator that increases cellular proliferation (9). YAP is also required for skin wound healing in normal mice (12). Therefore, the current study investigated the effects of SP administration on YAP expression in wounds. SP significantly increased the number of PCNA-positive proliferating cells in the dermis of $\mathrm{db} / \mathrm{db}$ mice compared with control mice $(32.3 \pm 1.60 \%$ vs. $3.30 \pm 0.46 \%$; Fig. 2A and B). In addition, YAP was detected in a significantly higher percentage of dermal cell nuclei in the SP-treated mice compared with control mice $(7.47 \pm 1.52 \%$ vs. $2.06 \pm 0.43 \%$; Fig. 2C and D). Overall, the results indicate that subcutaneous administration of SP may promote accelerated wound healing by inducing YAP-mediated cellular proliferation.

SP enhances angiogenesis in dermal wounds and induces mobilization of EPCs. A previous study demonstrated that subcutaneous SP treatment increases angiogenesis and the mobilization of EPCs in normal mice (17). In the present study, subcutaneous SP injection significantly increased the frequency of CD31-positive cells in the wounded dermis of $\mathrm{db} / \mathrm{db}$ mice, compared with the control (control, $1.77 \pm 0.35, \mathrm{n}=4 \mathrm{vs.} \mathrm{SP-treated,}$ $5.33 \pm 1.20$, n=4; Fig, 3A and B). EPC mobilization is important for angiogenesis and wound healing $(8,24)$, and its function is impaired in diabetes (8). In order to confirm the mobilization of EPCs, Matrigel plug assays were performed in uninjured $\mathrm{db} / \mathrm{db}$ mice, and subcutaneous SP treatment significantly increased the number of CD31-positive cells in uninjured $\mathrm{db} / \mathrm{db}$ mice by

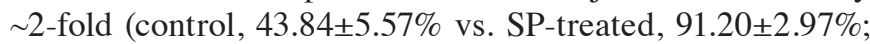
Fig. 4A and B). In addition to the SP-mediated increase in EPC mobilization, SP treatment also resulted in a 2-fold increase in the number of EPC-CFUs in the bone marrow of $\mathrm{db} / \mathrm{db}$ mice (control, 30.75 $\pm 5.07, \mathrm{n}=2$ vs. SP-treated, 62.00 $\pm 8.82, \mathrm{n}=3$; Fig. 4C). Therefore, subcutaneous treatment with SP may increase EPC mobilization, which may contribute to enhanced angiogenesis in the wound dermis and an increase in the EPC population within the bone marrow of $\mathrm{db} / \mathrm{db}$ mice.

\section{Discussion}

The present study demonstrated that subcutaneous administration of SP accelerated diabetic wound healing. Furthermore, it was also observed that subcutaneous injection of SP increased EPC mobilization in diabetes and increased the activation of YAP in the wound dermis of $\mathrm{db} / \mathrm{db}$ mice. Increased EPC mobilization and YAP activation may contribute to accelerated diabetic wound healing through enhanced angiogenesis and increased fibroblast proliferation, respectively. The results of a previous study (17) and the current study demonstrated that systemic SP treatment by subcutaneous injection induced EPC mobilization in normal and diabetic acute wounds. Notably, diabetic mice treated with SP exhibited a similar rate of wound closure compared with the normal wound healing process in normal mice (data not shown). However, the efficiency of EPC mobilization mediated by subcutaneous SP injection in the diabetic and normal wound environments was not compared. Furthermore, the mechanisms responsible for SP-mediated mobilization of EPCs remain undefined, and it is not established whether EPC migration in $\mathrm{db} / \mathrm{db}$ mice is mediated by the same mechanisms involved in normal acute wound healing. 
A

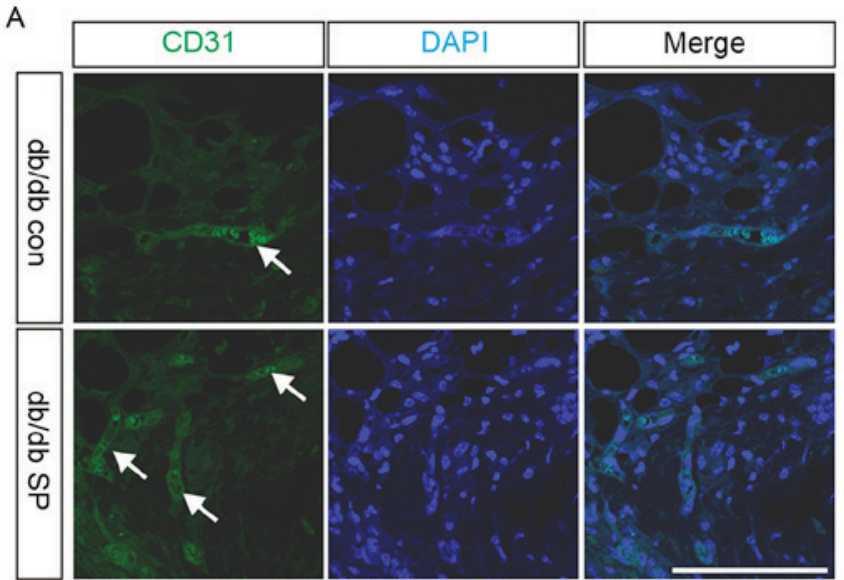

B

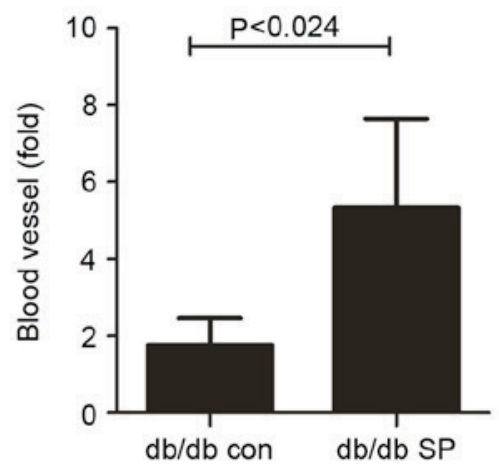

Figure 3. SP-induced angiogenesis in $\mathrm{db} / \mathrm{db}$ mice wounds. (A) Immunohistochemistry analysis of CD31 (green). Nuclei were stained in blue with DAPI. White arrows indicate CD31-positive signals in the wounds of $\mathrm{db} / \mathrm{db}$ con and $\mathrm{db} / \mathrm{db}$ SP mice. Scale bar, $100 \mu \mathrm{m}$. (B) Density of blood vessels in the wounds presented as a fold-change value. The number of blood vessels in a random image from a tissue section in the center of the wound was counted. The total area of the tissue in the analyzed image was used as the internal control for the number of blood vessels. Data for $\mathrm{db} / \mathrm{db}$ con $(\mathrm{n}=4)$ and $\mathrm{db} / \mathrm{db} \mathrm{SP}(\mathrm{n}=4)$ mice are presented as the mean + standard deviation. SP, substance $\mathrm{P} ; \mathrm{db} / \mathrm{db}$ con, control diabetic mice; $\mathrm{db} / \mathrm{db} \mathrm{SP}, \mathrm{SP}$-treated diabetic mice.

Further studies are required to address these questions. In the diabetic wound dermis, subcutaneous treatment with SP not only increased the number of cells expressing nuclear YAP, but also increased the number of proliferating cells. In addition, the number of $\alpha$ SMA-positive cells was increased in the diabetic wound dermis. Collectively, YAP activation, as a result of subcutaneous administration of SP, may lead to the upregulation of $\alpha$ SMA-positive cell proliferation, which may subsequently accelerate wound contracture. Notably, primary cultured dermal fibroblasts of $\mathrm{db} / \mathrm{db}$ mice expressed lower levels of YAP mRNA compared with normal mice (data not shown). Therefore, it is important to determine whether SP controls only YAP activation through post-translational regulatory processes, or if it influences YAP activation and its transcriptional level activity.

\section{Acknowledgements}

The current study was supported by Basic Science Research Program through the National Research Foundation of Korea funded by the Ministry of Education (grant

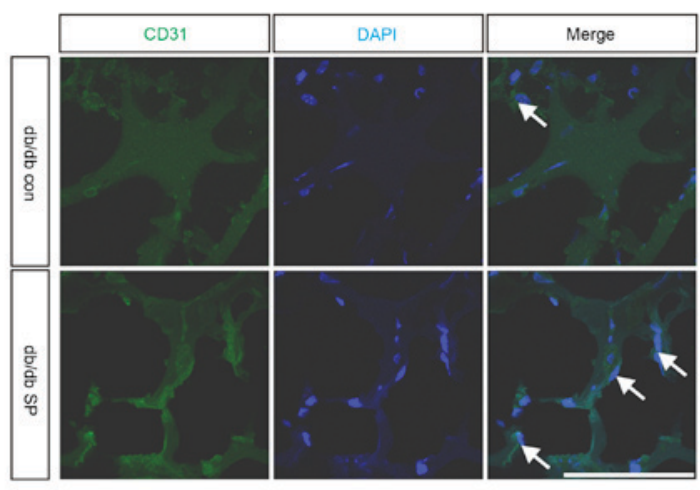

B
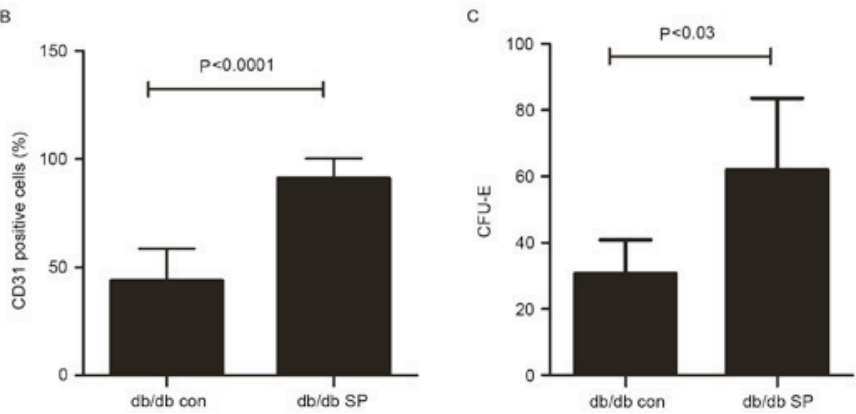

Figure 4. SP-mobilized EPCs and increased the number of EPC-CFUs from bone marrow. (A) CD31 immunostaining of the Matrigel plugs from $\mathrm{db} / \mathrm{db}$ con and $\mathrm{db} / \mathrm{db}$ SP groups. CD31 stained green and DAPI was used to stain the nuclei blue. White arrows indicate CD31-positive cells. Scale bar, $100 \mu \mathrm{m}$. (B) Density of CD31-positive cells in the Matrigel plugs was determined. The number of CD31-positive cells per section of the plug was counted and the total area of the analyzed plug section was used as an internal control for the total number of CD31-positive cells. Results are presented as the mean + standard deviation. (C) Number of EPC-CFUs from the bone marrow in $\mathrm{db} / \mathrm{db}$ con and db/db SP groups. SP, substance P; EPCs, endothelial progenitor cells; EPC-CFUs, EPC-colony forming units; db/db con, control diabetic mice; db/db SP, SP-treated diabetic mice.

no. 2015R1D1A1A09057839), the Korean Health Technology R\&D Project (Ministry of Health \& Welfare, Republic of Korea; grant no. HI13C1479) and a grant from Kyung Hee University in 2016 (grant no. KHU-20160701).

\section{References}

1. Shaw TJ and Martin P: Wound repair at a glance. J Cell Sci 122: 3209-3213, 2009.

2. Gurtner GC, Werner S, Barrandon Y and Longaker MT: Wound repair and regeneration. Nature 453: 314-321, 2008.

3. Brem H and Tomic-Canic M: Cellular and molecular basis of wound healing in diabetes. J Clin Invest 117: 1219-1222, 2007.

4. Velazquez OC: Angiogenesis and vasculogenesis: Inducing the growth of new blood vessels and wound healing by stimulation of bone marrow-derived progenitor cell mobilization and homing. J Vasc Surg 45 (Suppl A): A39-A47, 2007.

5. Rafii S and Lyden D: Therapeutic stem and progenitor cell transplantation for organ vascularization and regeneration. Nat Med 9: 702-712, 2003.

6. Amadesi S, Reni C,Katare R,Meloni M, Oikawa A,Beltrami AP, Avolio E, Cesselli D, Fortunato O, Spinetti G, et al: Role for substance p-based nociceptive signaling in progenitor cell activation and angiogenesis during ischemia in mice and in human subjects. Circulation 125: 1774-1786, S1-S19, 2012.

7. Fadini GP, Miorin M, Facco M, Bonamico S, Baesso I, Grego F, Menegolo M, de Kreutzenberg SV, Tiengo A, Agostini $\mathrm{C}$ and Avogaro A: Circulating endothelial progenitor cells are reduced in peripheral vascular complications of type 2 diabetes mellitus. J Am Coll Cardiol 45: 1449-1457, 2005. 
8. Gallagher KA, Liu ZJ, Xiao M, Chen H, Goldstein LJ, Buerk DG, Nedeau A, Thom SR and Velazquez OC: Diabetic impairments in NO-mediated endothelial progenitor cell mobilization and homing are reversed by hyperoxia and SDF-1 alpha. J Clin Invest 117: 1249-1259, 2007.

9. Yu FX and Guan KL: The Hippo pathway: Regulators and regulations. Genes Dev 27: 355-371, 2013.

10. Gumbiner BM and Kim NG: The Hippo-YAP signaling pathway and contact inhibition of growth. J Cell Sci 127: 709-717, 2014.

11. Hansen CG, Moroishi T and Guan KL: YAP and TAZ: A nexus for Hippo signaling and beyond. Trends Cell Biol 25: 499-513, 2015.

12. Lee MJ, Ran Byun M, Furutani-Seiki M, Hong JH and Jung HS: YAP and TAZ regulate skin wound healing. J Invest Dermatol 134: 518-525, 2014.

13. Hong HS, Lee J, Lee E, Kwon YS, Lee E, Ahn W, Jiang MH, Kim JC and Son Y: A new role of substance $P$ as an injury-inducible messenger for mobilization of CD29(+) stromal-like cells. Nat Med 15: 425-435, 2009.

14. Kant V, Kumar D, Kumar D, Prasad R, Gopal A, Pathak NN Kumar P and Tandan SK: Topical application of substance P promotes wound healing in streptozotocin-induced diabetic rats. Cytokine 73: 144-155, 2015.

15. Leal EC, Carvalho E, Tellechea A, Kafanas A, Tecilazich F, Kearney C, Kuchibhotla S, Auster ME, Kokkotou E, Mooney DJ, et al: Substance p promotes wound healing in diabetes by modulating inflammation and macrophage phenotype. Am J Pathol 185: 1638-1648, 2015.

16. Yang L, Di G, Qi X, Qu M, Wang Y, Duan H, Danielson P, Xie L and Zhou Q: Substance P promotes diabetic corneal epithelial wound healing through molecular mechanisms mediated via the neurokinin-1 receptor. Diabetes 63: 4262-4274, 2014.
17. Um J, Jung N, Chin S, Cho Y, Choi S and Park KS: Substance $P$ enhances EPC mobilization for accelerated wound healing. Wound Repair Regen 24: 402-410, 2016.

18. Wang LH, Zhou SX, Li RC, Zheng LR, Zhu JH, Hu SJ and Sun YL: Serum levels of calcitonin gene-related peptide and substance $\mathrm{P}$ are decreased in patients with diabetes mellitus and coronary artery disease. J Int Med Res 40: 134-140, 2012.

19. Antezana M, Sullivan SR, Usui M, Gibran N, Spenny M, Larsen J, Ansel J, Bunnett $\mathrm{N}$ and Olerud J: Neutral endopeptidase activity is increased in the skin of subjects with diabetic ulcers. J Invest Dermatol 119: 1400-1404, 2002.

20. Baustian C, Hanley S and Ceredig R: Isolation, selection and culture methods to enhance clonogenicity of mouse bone marrow derived mesenchymal stromal cell precursors. Stem Cell Res Ther 6: 151, 2015.

21. Hinz B: Formation and function of the myofibroblast during tissue repair. J Invest Dermatol 127: 526-537, 2007.

22. Darby IA, Bisucci T, Hewitson TD and MacLellan DG: Apoptosis is increased in a model of diabetes-impaired wound healing in genetically diabetic mice. Int J Biochem Cell Biol 29: 191-200, 1997.

23. Harvey KF, Zhang $X$ and Thomas DM: The Hippo pathway and human cancer. Nat Rev Cancer 13: 246-257, 2013.

24. Asahara T, Murohara T, Sullivan A, Silver M, van der Zee R, Li T, Witzenbichler B, Schatteman G and Isner JM: Isolation of putative progenitor endothelial cells for angiogenesis. Science 275: 964-967, 1997. 